\title{
Appearance of remodelled and dendritic cell-rich alveolar-lymphoid interfaces provides a structural basis for increased alveolar antigen uptake in chronic obstructive pulmonary disease
}

\author{
Michiko Mori, ${ }^{1}$ Cecilia K Andersson, ${ }^{2}$ Kaj A Svedberg, ${ }_{1}^{1}$ Pernilla Glader, ${ }^{2,3}$ \\ Anders Bergqvist, ${ }^{2}$ Medya Shikhagaie, ${ }^{1}$ Claes-Göran Löfdahl, ${ }^{2}$ Jonas S Erjefältt,
}

- Additional material is published online only. To view please visit the journal online (http://dx.doi.org/10.1136/ thoraxjnl-2012-202879)

${ }^{1}$ Department of Experimental Medical Science, Lund University, Lund, Sweden ${ }^{2}$ Department of Respiratory Medicine and Allergology, Lund University Hospital, Lund, Sweden

${ }^{3}$ Respiratory Medicine and Allergology at Sahlgrenska Academy, University of Gothenburg, Gothenburg, Sweden

\section{Correspondence to} Dr Jonas S Erjefält, Head, Unit of Airway Inflammation and Immunology, Department of Experimental Medical Science, BMC D12, Klinikgatan 30, Lund University,

Lund SE-22184, Sweden; Jonas.Erjefalt@med.lu.se

Received 16 October 2012 Revised 6 January 2013 Accepted 16 January 2013 Published Online First 14 February 2013
To cite: Mori M, Andersson CK, Svedberg KA, et al. Thorax 2013:68:521531.

\section{ABSTRACT}

Rationale The alveolar pathology in chronic obstructive pulmonary disease (COPD) involves antigen-driven immune events. However, the induction sites of alveolar adaptive immune responses have remained poorly investigated.

Objectives To explore the hypothesis that interfaces between the alveolar lumen and lymphoid aggregates (LAs) provide a structural basis for increased alveolar antigen uptake in COPD lungs.

Methods Lung samples from patients with mild (Global Initiative for Chronic Obstructive Lung Disease (GOLD) stage I), moderate-severe (GOLD II-III), and very severe (GOLD IV) COPD were subjected to detailed histological assessments of adaptive immune system components. Never smokers and smokers without COPD served as controls.

Results Quantitative histology, involving computerised three-dimensional reconstructions, confirmed a rich occurrence of alveolar-restricted LAs and revealed, for the first time, that the vast majority of vascular or bronchiolar associated LAs had alveolar interfaces but also an intricate network of lymphatic vessels. Uniquely to COPD lungs, the interface epithelium had transformed into a columnar phenotype. Accumulation of langerin $(C D 207)^{+}$dendritic cells occurred in the interface epithelium in patients with COPD but not controls. The antigen-capturing capacity of langerin ${ }^{+}$dendritic cells was confirmed by increased alveolar protrusions and physical T cell contact. Several of these immune remodelling parameters correlated with lung function parameters.

Conclusions Severe stages of COPD are associated with an emergence of remodelled and dendritic cell-rich alveolar-lymphoid interfaces. This novel type of immune remodelling, which predicts an increased capacity to respond to alveolar antigens, is suggested to contribute to aggravated inflammation in COPD.

\section{INTRODUCTION}

Chronic obstructive pulmonary disease (COPD) is one of the leading causes of morbidity and mortality worldwide. $^{1}$ Long-term exposure to inhaled chemical irritants in tobacco smoke results in a chronic and destructive inflammation in the lung. ${ }^{3}$ Apart from innate immune responses in COPD, it is becoming increasingly clear that

\section{Key messages}

What is the key question?

- Can peripheral lymphocyte-rich lymphoid aggregates in patients with chronic obstructive pulmonary disease (COPD) provide a structural basis for alveolar antigen uptake in the peripheral lung?

\section{What is the bottom line?}

- Severe stages of COPD are associated with remodelling of the interfaces between the alveolar lumen and lymphoid aggregates. These changes, which include a selective accumulation of dendritic cells to the interface epithelium, correlate with lung function and are suggested to increase the lung's capacity to respond to alveolar immune triggers.

\section{Why read on?}

- This novel type of structural alteration is likely to contribute to the induction of adaptive immune responses in patients with advanced stages of COPD and may also represent $a$ general adaptation to facilitate antigen uptake in the distal lung.

antigen-driven adaptive immune responses are activated in COPD lungs. ${ }^{4}$

In conducting airways, epithelial dendritic cells (DCs) extend cytoplasmic protrusions to sample luminal antigens. ${ }^{5}$ When an antigen is encountered, DCs migrate to draining lymph nodes and present the antigen to naïve $\mathrm{T}$ cells, thus initiating adaptive immune responses. ${ }^{6}$ Importantly, adaptive immune responses in COPD also take place locally within the lungs as demonstrated by the presence of bronchiolar-associated lymphoid aggregates. ${ }^{7}$ Since only scarce numbers of such lymphoid aggregates (LAs) are present in healthy subjects, these structures are thought to develop as a result of extended periods of inflammation or infections (ie, lymphoid neogenesis). ${ }^{8-10}$ Consequently lung LAs are not regarded as an integral part of secondary lymphoid organs (eg, lymph nodes or Peyer's patches in the 
intestines) and are therefore called tertiary lymphoid organs or ectopic lymphoid tissues. ${ }^{10}$

Lung LAs may acquire inhaled luminal antigens via migration of superficial airway DCs. ${ }^{11}$ However, LAs have also been observed at sites distant from the conducting airways. ${ }^{12-14}$ It remains unclear whether or not these structures acquire luminal antigens from other lung regions such as the alveolar parenchyma, a compartment that in COPD is frequently subjected to inflammation and infections. ${ }^{15}$ However, although alveolar adaptive immune responses are likely to occur extensively in COPD, little is known about how and where the alveolar antigen uptake takes place.

Recent experiments in mice show that when antigen is instilled into the lungs, most of the total antigen uptake is carried out by scattered DCs in the alveolar parenchyma. ${ }^{16} 17$ However, it can be speculated that in human airway diseases, such as COPD, alveolar antigen uptake is also carried out at specialised alveolar-lymphoid interfaces, similar to the luminallymphoid interfaces in classical mucosal-associated lymphoid tissues. However, despite such an arrangement facilitates a localised and more rapid antigen presentation, alveolar-lymphoid interfaces have never been explored previously. This study performs the first focused exploration of this interface and tests the hypothesis that DC-rich interfaces between the alveolar lumen and LAs provide a structural basis for increased alveolar antigen uptake in COPD.

\section{METHODS}

Additional methodological details are provided in the online supplementary data.

\section{Patients}

Lung tissue from patients with COPD and never-smoking and smoking controls was collected from 46 subjects undergoing lung transplantation or surgical resection for suspected lung cancer. ${ }^{18}$ The patients with COPD were divided into mild (Global Initiative for Chronic Obstructive Lung Disease (GOLD) stage I), moderate to severe (GOLD II-III) and very severe (GOLD IV) COPD. The subject characteristics, inclusion criteria and methodological details, are presented in table 1 and in the online supplementary data.

All subjects gave written informed consent to participate in the study, which was approved by the local ethics committee.

\section{Characterisation of peripheral lung LAs}

For each subject multiple peripheral lung samples were subjected to standardised fixation and processing for generating paraffin sections. LAs, defined as more than 50 contiguous lymphoid cells, were quantified and subgrouped according to their anatomical location: small airway/bronchiolar-associated lymphoid tissue (abbreviated BRALT in order to make a distinction to bronchial-associated lymphoid tissue BALT), vascular-associated lymphoid tissue (VALT, defined as LAs in non-bronchiolar-associated solitary vessels with a diameter $>140 \mu \mathrm{m}$ ) and alveolar-only lymphoid tissue (ALT, defined as LAs well separated from any bronchioles, pleural tissue or pulmonary vessels).

\section{Immunohistochemistry}

Immunohistochemistry (IHC) was performed in an automated IHC robot (DakoCytomation, Glostrup, Denmark). All primary antibodies used (see table 2) and IHC protocols have been validated extensively for use on paraffin sections.

The immunoreactivity was visualised using the Dako EnVision kit. ${ }^{19}$ For bright field double staining the first primary antibody was detected with horseradish peroxidase-conjugated antibodies and the second with alkaline phosphatase-conjugated

Table 1 Subject characteristics

\begin{tabular}{|c|c|c|c|c|c|}
\hline Characteristic & Never smokers & Smokers w/o COPD & GOLD I COPD & GOLD II-III* COPD & GOLD IV COPD \\
\hline Subjects, $n$ & 8 & 7 & 6 & 15 & 10 \\
\hline Gender, men/women & $2 / 6$ & $3 / 4$ & $4 / 2$ & $12 / 3$ & $4 / 6$ \\
\hline Age, years & $66(33-76) \dagger$ & $56(47-68)$ & $68(56-75)$ & $67(53-77)$ & $62(53-66)$ \\
\hline Smoking history, pack-years & 0 & $36(20-80)$ & $39(25-66)$ & $46(16-65)$ & $43(25-60)$ \\
\hline Smoking status, ex smokers/current & - & $3 / 4$ & $3 / 3$ & $10 / 5$ & $10 / 0$ \\
\hline $\mathrm{FEV}_{1}$ & $2.5(1.7-5.1)$ & $3.0(1.9-3.5)$ & $2.9(1.6-3.2)$ & $1.9(1.2-2.9)$ & $0.6(0.4-1.0)$ \\
\hline $\mathrm{FEV}_{1}, \%$ of predicted & $108.9(82-141)$ & $95.0(82-120)$ & $85.9(80-95)$ & $68.5(43-78)$ & $23.1(15-27)$ \\
\hline $\mathrm{FEV}_{1} /(\mathrm{F}) \mathrm{VC}, \%$ & $81.5(66-121)$ & $76.0(71-88)$ & $66.5(65-70)$ & $58.0(41-68)$ & $32.0(20-39)$ \\
\hline \multicolumn{6}{|l|}{ Inhaled $\beta_{2}$ agonists } \\
\hline Short acting (yes/no/unknown) & $0 / 8 / 0$ & $0 / 7 / 0$ & $1 / 5 / 0$ & $2 / 13 / 0$ & $4 / 5 / 1 \ddagger$ \\
\hline Long acting (yes/no/unknown) & $0 / 8 / 0$ & $0 / 7 / 0$ & $0 / 6 / 0$ & $0 / 15 / 0$ & $4 / 5 / 1 \ddagger$ \\
\hline \multicolumn{6}{|l|}{ Inhaled anticholinergics } \\
\hline Short acting (yes/no/unknown) & $0 / 8 / 0$ & $0 / 7 / 0$ & $1 / 5 / 0$ & $2 / 13 / 0$ & $3 / 6 / 1 \neq$ \\
\hline Long acting (yes/no/unknown) & $0 / 8 / 0$ & $0 / 7 / 0$ & $0 / 6 / 0$ & $0 / 15 / 0$ & $5 / 4 / 1 \neq$ \\
\hline Inhaled short acting $\beta_{2}$ agonist plus anticholinergics (yes/no/unknown) & $0 / 8 / 0$ & $0 / 7 / 0$ & $0 / 6 / 0$ & $0 / 15 / 0$ & $3 / 6 / 1 \ddagger$ \\
\hline \multicolumn{6}{|l|}{ Corticosteroids } \\
\hline Inhaled (yes/no/unknown) & $0 / 8 / 0$ & $0 / 7 / 0$ & $0 / 6 / 0$ & $1 / 14 / 0$ & $2 / 7 / 1 \ddagger$ \\
\hline Oral (yes/no/unknown) & $0 / 8 / 0$ & $0 / 7 / 0$ & $0 / 6 / 0$ & $0 / 15 / 0$ & $2 / 7 / 1 \ddagger$ \\
\hline Inhaled long acting $\beta_{2}$ agonist plus corticosteroids (yes/no/unknown) & $0 / 8 / 0$ & $0 / 7 / 0$ & $0 / 6 / 0$ & $1 / 14 / 0$ & $7 / 2 / 1 \ddagger$ \\
\hline Mucolytics (yes/no/unknown) & $0 / 8 / 0$ & $0 / 7 / 0$ & $2 / 4 / 0$ & $0 / 15 / 0$ & $5 / 4 / 1 \neq$ \\
\hline
\end{tabular}


Table 2 Primary antibodies used for immunohistochemistry

\begin{tabular}{|c|c|c|c|c|c|}
\hline Antigen & Clone* & Supplier & Antigen retrieval & Dilutiont & Against \\
\hline CD20 & $\mathrm{L} 26$ & Dako, Glostrup, Denmark & Low pH PT & $\begin{array}{l}1: 1200 \\
1: 600 \text { (IF) }\end{array}$ & B cells \\
\hline CD4 & $1 F 6$ & Novocastra ${ }^{\S}$, Newcastle Upon Tyne, UK & Low pH‡ PT & $1: 50$ & T-helper cells \\
\hline CD11c & $5 \mathrm{D} 11$ & Novocastra ${ }^{\S}$ & Low pH‡ PT & $1: 100$ & Macrophages and myeloid dendritic cells \\
\hline CD68 & PG-M1 & Dako & Low pH‡ PT & $1: 500$ & Monocytes and macrophages \\
\hline CD21 & 1F8 & Dako & Low pHף PC & $1: 100$ & Follicular dendritic cells \\
\hline BDCA-2 & $104 C 12.08$ & Dendritics, Lyon, France & Low $\mathrm{pH}^{* *} \mathrm{PC}$ & $1: 50$ & Plasmacytoid dendritic cells \\
\hline Multi-Cytokeratin & AE1/AE3 & Novocastra ${ }^{\S}$ & Low pH PT & $\begin{array}{l}1: 300 \\
1: 150 \text { (IF) }\end{array}$ & Epithelial cells \\
\hline Ki-67 & Rabbit polyclonal & Biocare Medical, Concord, CA, USA & Low $\mathrm{pH}^{* *} \mathrm{PC}$ & $1: 200$ & Cell proliferation \\
\hline Langerin (CD207) & 12D6 & Novocastra ${ }^{\S}$ & Low pH‡ PT & $\begin{array}{l}1: 100 \\
1: 80 \text { (IF) }\end{array}$ & Langerhans-like dendritic cells \\
\hline Podoplanin & D2-40 & Biocare Medical & Low $\mathrm{pH}^{* *} \mathrm{PC}$ & $1: 100$ & Lymphatic endothelium \\
\hline$\alpha$-Smooth muscle actin & $1 \mathrm{~A} 4$ & Sigma-Aldrich, St Louis, MO, USA & Low $\mathrm{pH}^{* *} \mathrm{PC}$ & $1: 1000$ & Smooth muscle cells \\
\hline
\end{tabular}

antibodies. Immunofluorescence double staining was performed to evaluate colocalisation. ${ }^{18}$

\section{Serial section-based three-dimensional reconstruction of LAs}

A series of serial sections $(>30)$ was used to generate image segmentation data of LAs, parenchymal tissue and immunostained lymphatic vessels. Structure-specific segmentation data were loaded into a software platform (Amira 5.4.2 Visage Imaging Inc, San Diego, California, USA) to reveal the three-dimensional structure.

\section{Quantifications}

For each subject multiple large sections (from two to three separated peripheral lung regions) were analysed. High-resolution digital images, representing the entire section area, were generated from all bright field sections using a slide-scanning robot (ScanScope, Aperio Technologies, Vista, California, USA). Morphometric analyses and quantification of immunoreactivity were performed using Aperio ImageScope V.10.0 software (Aperio Technologies). Apart from multiple alveolar regions, a total of around 1000 individual bronchioles and pulmonary vessels were evaluated. Alveolar-LA interfaces were measured by manual cursor tracing. Langerin ${ }^{+}$DCs were manually counted along alveolar-LA interfaces and in distinct regions within the LAs. Quantifications involving manual counting or cursor tracing were performed on blinded sections.

\section{Statistics}

Values are given as median (range), unless otherwise stated. The Kruskal-Wallis non-parametric test followed by Dunn's multiple comparisons post test was used for comparison between study groups and the Mann-Whitney test was used for comparisons between two groups (Prism V.5.0, GraphPad Software, San Diego, California, USA). Spearman's rank method was used to calculate correlation coefficients.

\section{RESULTS}

LAs are present in all major peripheral lung compartments

Two-dimensional approach to assess the distribution of LAs

Prompted by initial observations of a widespread anatomical distribution of LAs in COPD lungs (see online supplementary figure S1 and table S1), all LAs were quantified and divided into BRALTs, VALTs and ALTs (figure 1A, see online supplementary figure S1 and table S1). The BRALT structures were mainly observed in the adventitia layer of the bronchioles. Across all study groups an average of $17.7 \%$ of the BRALTs reached the bronchiolar epithelium. Although the percentage of bronchioles containing BRALTs was increased in GOLD stage IV COPD compared with never smokers $(p<0.05)$, the number of BRALTs was not changed among the study groups. The number of VALTs, which had an almost exclusive adventitial distribution, increased in patients with GOLD stage IV COPD compared with never-smoking control subjects $(\mathrm{p}<0.01)$. In the alveolar parenchyma, patients with COPD displayed a tendency to increased number of ALTs (see online supplementary table S1). In GOLD stage IV, most LAs were well developed as indicated by Ki- $67^{+}$proliferating cells and increased CD $21^{+}$follicular DCs (see online supplementary data). Follicular CD21 immunoreactivity was observed equally frequently among BRALTs, VALTs and ALTs.

\section{Three-dimensional confirmation of true alveolar-only LAs}

A serial section-based three-dimensional analysis was performed to reveal the true spatial distribution of the lung LAs. This analysis confirmed the presence of true ALT structures; that is, alveolar-only LAs without physical contact with bronchioles or pulmonary vessels (figure 1B-D). In COPD, an average of $25.4 \%$ of all LAs were true ALTs and $31.2 \%$ of all LAs were VALTs. Consequently, the remaining $43.4 \%$ of the LAs were BRALTs. This serial section analysis also revealed that $79.8 \%$ of all identified ALTs in two-dimensional sections were in fact true ALTs. Similarly, $89.3 \%$ of all VALTs were true VALTs.

\section{BRALT and VALT structures have extensive interfaces} with the alveolar lumen and the lymphatics

The vast majority of the LAs, including BRALTs and VALTs, had a direct interface towards the alveolar lumen (figure 2A,C,D). 
A Anatomical distribution
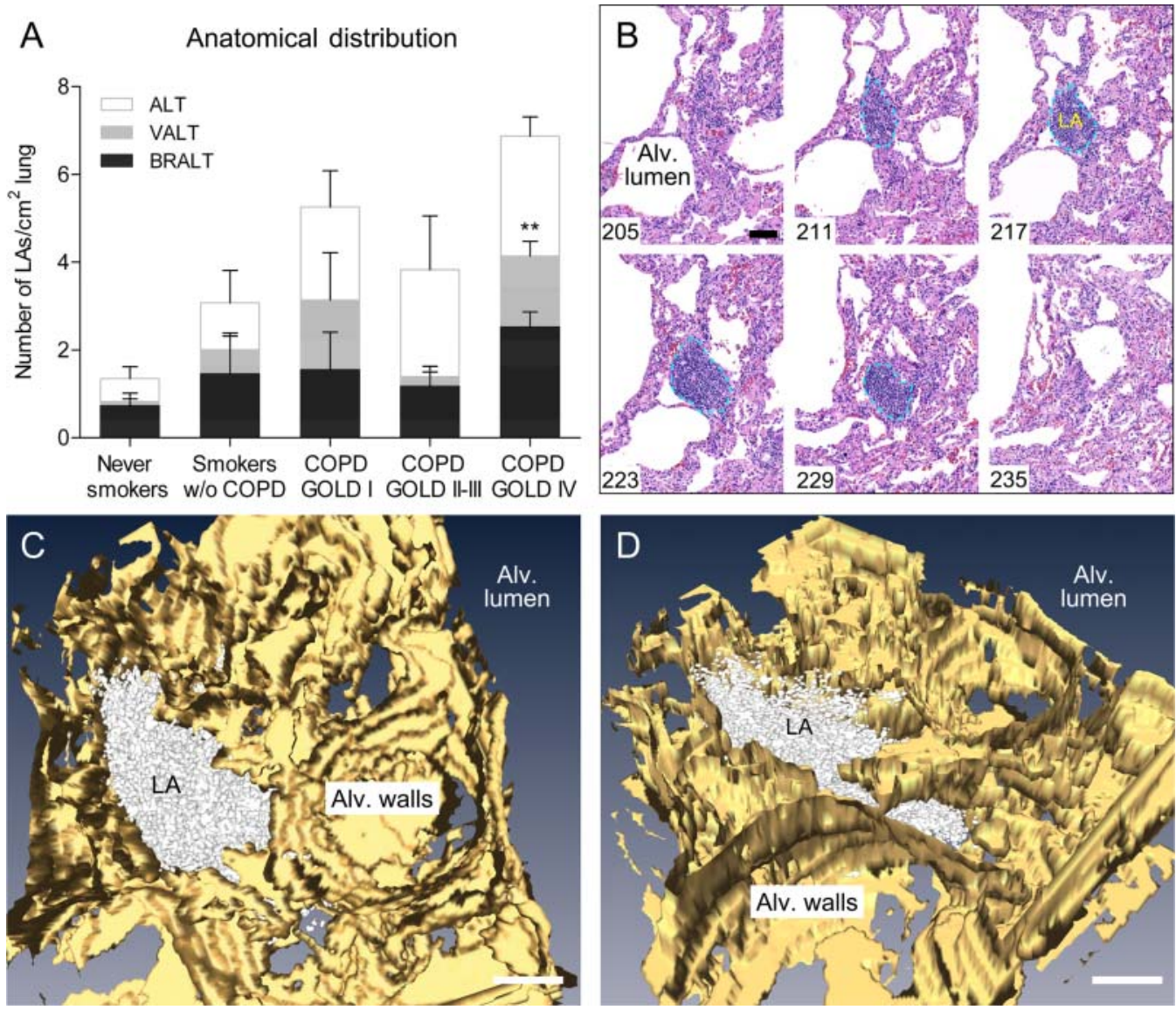

Figure 1 Lymphoid aggregates (LAs) are formed in all major lung compartments of the peripheral lung. (A) Bar graph showing the relative proportion of alveolar-only lymphoid tissue (ALT), vascular-associated lymphoid tissue (VALT), and bronchiolar-associated lymphoid tissue (BRALT) structures, as revealed by two-dimensional analysis of Masson's trichrome-stained sections from never smokers, smokers without chronic obstructive pulmonary disease (COPD) and patients with Global Initiative for Chronic Obstructive Lung Disease (GOLD) stage I-IV COPD. Values are given as mean \pm SEM. Statistical analysis was performed using the Kruskal-Wallis non-parametric test followed by Dunn's multiple comparison post test. ${ }^{* *} p<0.01$. (B) Examples from a series with $>400$ haematoxylin-eosin stained sections ( $4 \mu \mathrm{m}$ thick) used to reveal the true three-dimensional distribution of peripheral lung LAs. The images exemplify the gradual emergence and disappearance of a LA (outlined by dashed lines) without any associated pulmonary vessel or conducting airway. The section number is indicated in the images. (C) Similar image stacks were used to visualise true alveolar LA by computerised three-dimensional reconstruction, exemplified by a LA (white) and surrounding parenchymal tissue (yellow) and alveolar lumen (blue grey) (see online supplementary data for methodological details). (D) Three-dimensional image of another viewing angle. Scale bars: (B) $100 \mu \mathrm{m}$; (C,D) $200 \mu \mathrm{m}$

Irrespective of anatomic location, no difference in the frequency of LAs with alveolar interfaces was found between the study groups (figure $2 \mathrm{~A}$ and table 3 ).

The total length of alveolar interfaces, which is the combination of LA numbers and mean length of alveolar interfaces, was increased in advanced COPD compared with never-smoking controls (figure 2B). Among all LAs and study groups, an average of $33.4 \%$ of the LA perimeter was facing alveolar lumen (figure $2 \mathrm{~F}$ ).

Immunohistochemical visualisation of podoplanin ${ }^{+}$lymphatic vessels demonstrated a close spatial relationship between LAs and lymphatics (figure 2C,D). Three-dimensional reconstruction of podoplanin-stained lymphatic vessels further revealed the intricate connection among BRALT, VALT, ALT and lymphatics (exemplified in figure 2E). Across all study groups lymphatic vessels comprised an average of $20.3 \%$ of the LA perimeter (figure 2F).

\section{Remodelling of alveolar-lymphoid interface epithelium in COPD}

In never-smoking controls the alveolar-lymphoid epithelium was, at all locations, primarily of a simple squamous type (figure 3A,E).
A similar epithelium, but with increasing stretches of cuboidal epithelium, was present in smokers and patients with COPD (figure 3B,F). At more advanced stages of COPD (GOLD II-IV) the occurrence of cuboidal epithelium was accompanied by a higher columnar, non-ciliated epithelium (figure $3 \mathrm{G}, \mathrm{H}$ ). The emergence of columnar epithelium occurred similarly among BRALTs, VALTs and ALTs (table 3 ) and resulted in a significantly increased proportion and increased total columnar epithelial length in patients with GOLD IV COPD (figure 3C,D). Importantly, the columnar epithelium in patients with GOLD II-IV COPD was restricted to alveolar-LA interfaces (similar epithelium was not found in surrounding lung parenchyma or along other alveolar interfaces facing, for example, the adventitia of pulmonary vessels or conducting airways).

\section{Selective accumulation of antigen-presenting DCs along alveolar-lymphoid interfaces in COPD lungs}

Langerin $(C D 207)^{+}$DCs represent a major antigen-presenting cell type in the conducting airways in COPD. ${ }^{20}{ }^{21}$ Hence, we explored if langerin ${ }^{+}$DCs were present at alveolar-LA interfaces. Irrespective of anatomic location, no or very few interface langerin $^{+}$DCs were present in never-smoking and smoking 
A
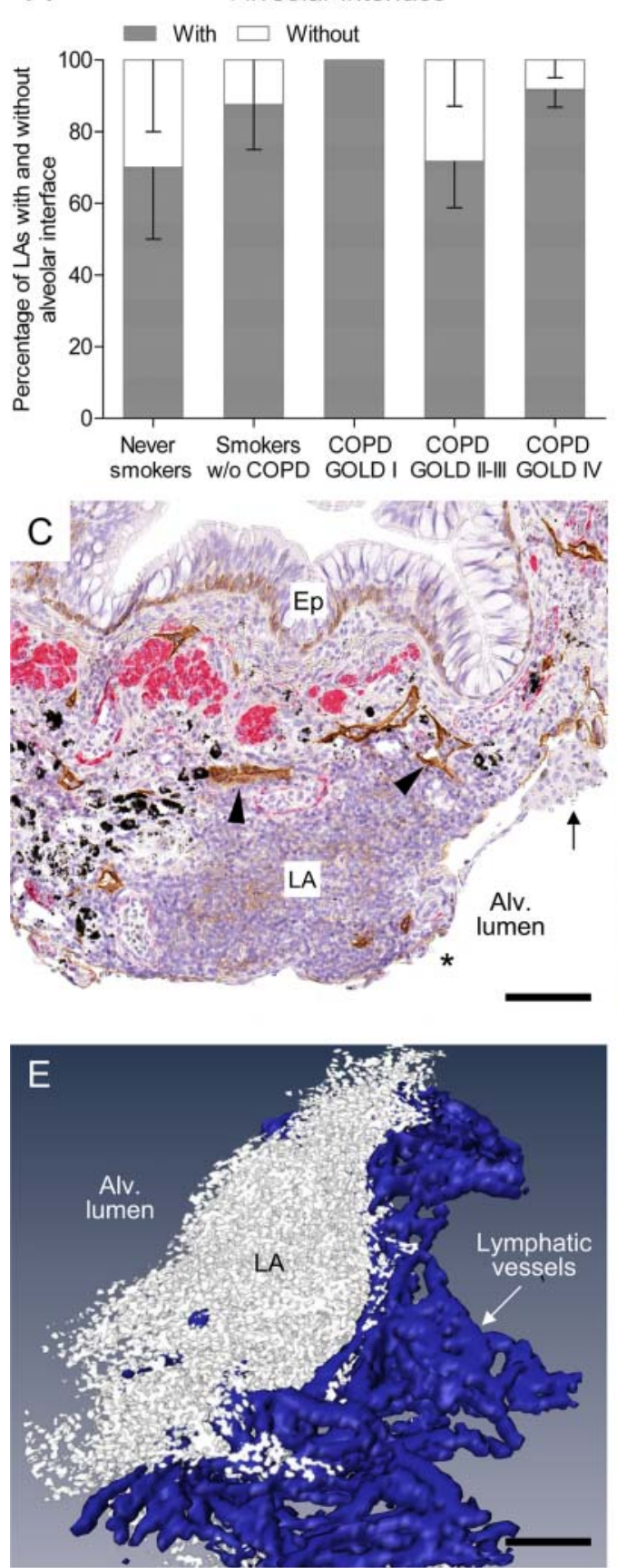

B Total length of alveolar interfaces
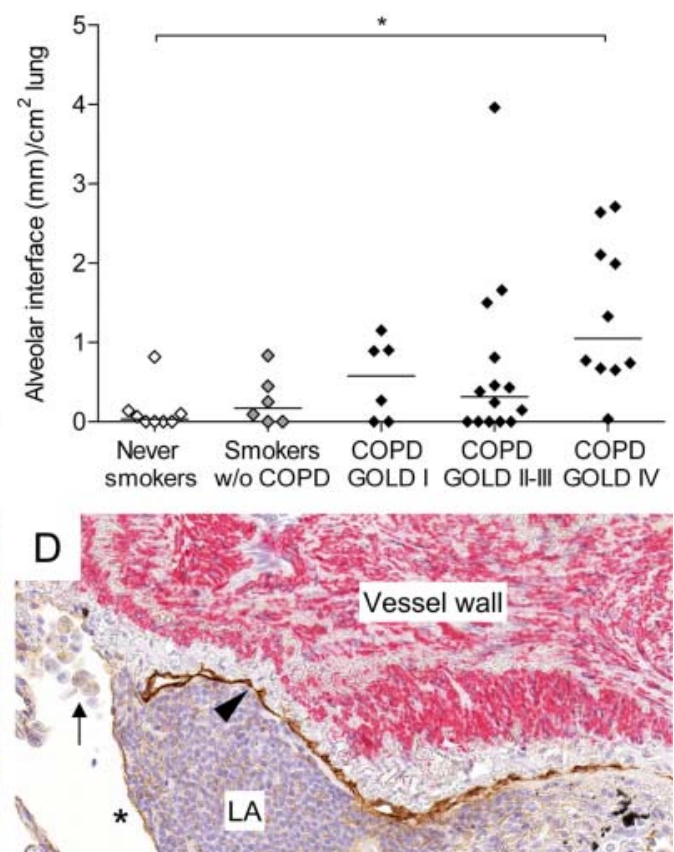

Alv.

lumen

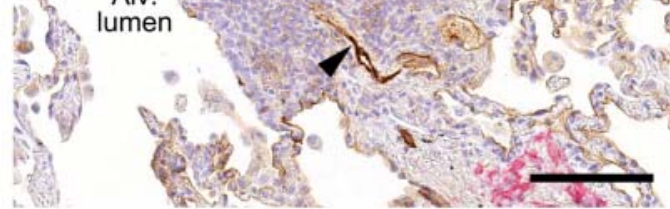

\section{F Proportion of surrounding structures}

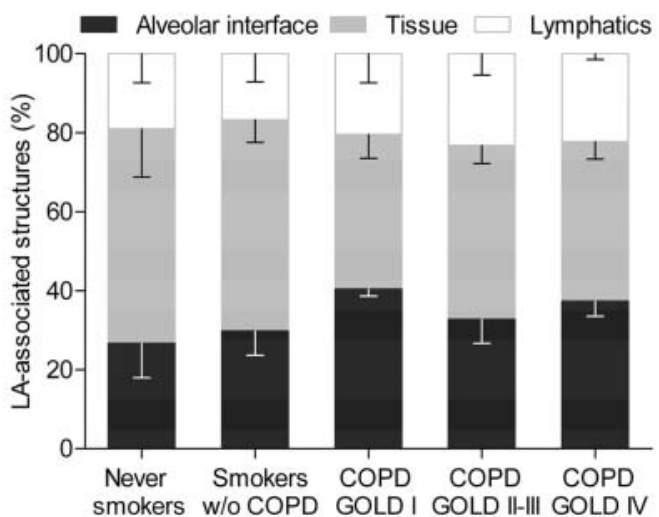

Figure 2 Lymphoid aggregates (LAs) have extensive interfaces with the alveolar lumen and peripheral lymphatic vessels. (A) Quantitative data showing that in all study groups the majority of LAs had an alveolar lumen-LA interface, irrespective of anatomical localisation. Values are given as mean \pm SEM. (B) Quantification of the total length of alveolar-LA interfaces. Statistical analysis was performed using the Kruskal-Wallis non-parametric test followed by Dunn's multiple comparison post test. Horizontal lines indicate medians for each group. * $\mathrm{p}<0.05$. (C) Photomicrograph of a typical bronchiolar-associated lymphoid tissue (BRALT) with its interface with the alveolar lumen (asterisk) and podoplanin ${ }^{+} /$ $\alpha$-smooth muscle actin ${ }^{+}$lymphatic vessels (arrowheads; brown). $\alpha$-Smooth muscle actin ${ }^{+}$cells are shown in red. Arrow indicates alveolar macrophages and 'Ep' the bronchiolar epithelium. Black endogenous pigment depositions are also visible. Epithelial basal cells and pneumocytes showed a weak immunoreactivity for podoplanin. Cell nuclei were counterstained with Mayer's haematoxylin (blue stain). (D) Photomicrograph of a similarly stained vascular-associated lymphoid tissue (VALT) with its direct interface with the alveolar lumen and extensive connection to podoplanin ${ }^{+} / \alpha$-smooth muscle actin ${ }^{+}$lymphatic vessel (arrowheads). (E) Three-dimensional rendering of an image stack with $40(4 \mu \mathrm{m}$ thick) serial sections revealed an intricate relationship between immunostained lymphatic vessels and peripheral lung LA. White: LA; blue: lymphatic vessels. (F) The relative proportion of anatomic structures along the LA perimeters, divided into alveolar interface, lymphatic vessel borders and remaining firm tissue (ie, airway wall tissue, adventitial tissue of pulmonary vessels, or parenchymal tissue). Values are given as mean \pm SEM. Scale bars: (C-E) $100 \mu \mathrm{m}$. 
Table 3 Characteristics of alveolar lumen-LA interfaces

\begin{tabular}{|c|c|c|c|c|c|c|}
\hline Parameters & Never smokers & Smokers w/o COPD & GOLD I COPD & GOLD II-III COPD & GOLD IV COPD & Overall $p$ value \\
\hline \multicolumn{7}{|c|}{ LAs with alveolar interfaces, \% } \\
\hline BRALT & $50(0-100)$ & $50(0-100)$ & $100(100-100)$ & $100(100-100)$ & $100(0-100)$ & 0.205 \\
\hline VALT & NA & $100(100-100)$ & $100(100-100)$ & $100(0-100)$ & $100(83-100)$ & 0.427 \\
\hline ALT & $100(100-100)$ & $100(100-100)$ & $100(100-100)$ & $100(75-100)$ & $100(100-100)$ & 0.615 \\
\hline \multicolumn{7}{|c|}{ Simple squamous epithelium, $\%$ of total alveolar interface } \\
\hline BRALT & $97(93-100)$ & $80(80-80)$ & $100(100-100)$ & $67(9-100)$ & $55(37-73)$ & 0.077 \\
\hline VALT & NA & $74(74-74)$ & $51(0-100)$ & $73(57-100)$ & $63(0-84)$ & 0.655 \\
\hline ALT & $100(84-100)$ & $71(53-89)$ & $61(10-70)$ & 77 (40-89) & $81(56-97)$ & 0.126 \\
\hline \multicolumn{7}{|c|}{ Simple cuboidal epithelium, $\%$ of total alveolar interface } \\
\hline BRALT & $3(0-7)$ & $20(20-20)$ & 0 & $17(0-91)$ & $26(13-41)$ & 0.115 \\
\hline VALT & NA & $26(26-26)$ & $49(0-100)$ & $25(0-43)$ & $20(13-100)$ & 0.724 \\
\hline ALT & $0(0-16)$ & $29(11-47)$ & $39(30-90)$ & $23(11-60)$ & $13(2-36)$ & $>0.050$ \\
\hline \multicolumn{7}{|c|}{ Columnar epithelium, $\%$ of total alveolar interface } \\
\hline BRALT & 0 & 0 & 0 & $0(0-65)$ & $17(0-40)$ & 0.097 \\
\hline VALT & NA & 0 & 0 & $0(0-6)$ & $6(0-68)$ & 0.141 \\
\hline ALT & 0 & 0 & 0 & 0 & $1(0-34)$ & 0.089 \\
\hline \multicolumn{7}{|c|}{ Number of langerin ${ }^{+} \mathrm{DCs}$ per $\mathrm{mm}^{2}$ of LA } \\
\hline All LAs & $87(19-262)$ & $50(31-128)$ & $141(90-262)$ & $113(0-203)$ & $147(85-228)$ & 0.225 \\
\hline BRALT & $38(37-104)$ & 0 & $151(141-161)$ & $94(0-218)$ & $130(108-254)$ & 0.036 \\
\hline VALT & NA & $70(31-190)$ & $174(61-288)$ & $179(27-232)$ & $145(96-409)$ & 0.508 \\
\hline ALT & $182(102-262)$ & $100(99-101)$ & $168(155-182)$ & $79(0-203)$ & $126(15-181)$ & 0.256 \\
\hline \multicolumn{7}{|c|}{ Number of langerin ${ }^{+} \mathrm{DCs}$ per $\mathrm{mm}$ of total alveolar interface } \\
\hline All LAs & 0 & $0.14(0-2.36)$ & $2.96(0-5.27)$ & $2.11(0-5.76)$ & $3.10(1.08-4.77)$ & 0.031 \\
\hline BRALT & 0 & NA & $0.83(0-1.67)$ & $0(0-3.39)$ & $3.03(0.95-5.16)$ & 0.061 \\
\hline VALT & NA & $0(0-3.82)$ & $5.11(3.21-7.02)$ & $8.56(0-11.86)$ & $4.18(1.03-8.22)$ & 0.411 \\
\hline ALT & 0 & $0.84(0.28-1.40)$ & $1.87(0-3.74)$ & $1.57(0-9.19)$ & $1.70(0-4.78)$ & 0.349 \\
\hline \multicolumn{7}{|c|}{ Number of langerin ${ }^{+} \mathrm{DCs}$ per $\mathrm{mm}$ of simple squamous interface } \\
\hline BRALT & 0 & NA & $0.54(0-1.07)$ & $0(0-3.46)$ & $0.74(0-3.96)$ & 0.382 \\
\hline VALT & NA & 0 & $0.24(0-0.47)$ & $0(0-6.81)$ & $0(0-2.86)$ & 0.592 \\
\hline ALT & 0 & 0 & $3.01(0-6.01)$ & $0(0-6.45)$ & $1.16(0-4.93)$ & 0.429 \\
\hline \multicolumn{7}{|c|}{ Number of langerin ${ }^{+}$DCs per $\mathrm{mm}$ of simple cuboidal interface } \\
\hline BRALT & 0 & NA & $1.89(0-3.78)$ & $0(0-6.52)$ & $1.70(0-6.29)$ & 0.211 \\
\hline VALT & NA & $0(0-5.89)$ & $9.78(5.81-13.74)$ & $5.66(0-26.06)$ & $6.85(0-15.71)$ & 0.523 \\
\hline ALT & 0 & $4.64(4.18-5.09)$ & 0 & $3.05(0-13.70)$ & $0.72(0-7.97)$ & 0.183 \\
\hline \multicolumn{7}{|c|}{ Number of langerin ${ }^{+}$DCs per $\mathrm{mm}$ of columnar interface } \\
\hline All LAs & NA & NA & NA & $5.26(3.97-6.56)$ & $2.10(0-9.48)$ & 0.178 \\
\hline BRALT & NA & NA & NA & $2.98(0-5.95)$ & $3.44(0-7.13)$ & 1.000 \\
\hline VALT & NA & NA & NA & $9.84(0-19.69)$ & $2.94(0-24.83)$ & 1.000 \\
\hline ALT & NA & NA & NA & 0 & $0(0-7.12)$ & 0.422 \\
\hline
\end{tabular}

Values are median (range).

${ }^{*}$ Kruskal-Wallis test for differences between groups or Mann-Whitney test for comparisons between two groups.

ALT, alveolar-only lymphoid tissue; BRALT, small airway/bronchiolar-associated lymphoid tissue; COPD, chronic obstructive pulmonary disease; DC, dendritic cell; GOLD, Global Initiative for Chronic Obstructive Lung Disease; LA, lymphoid aggregate; NA, not available; VALT, vascular-associated lymphoid tissue.

controls (figure 4D-F). In contrast, abundant and increased numbers of langerin ${ }^{+}$DCs were observed in COPD (figure 4D$\mathrm{F}$ and table 3). No, or few such DCs were present in non-LA alveolar interfaces.

A detailed analysis of the distribution of langerin ${ }^{+}$DCs in LAs with an alveolar interface revealed a selective accumulation of DCs to the alveolar interface region. The highest density was within the alveolar interface epithelium (figures 4A and 5). Also within the lymphoid tissue, the DCs accumulated to the superficial region just beneath the alveolar interface epithelium (figure 4G). In all COPD groups the superficial LA regions had significantly higher DC densities compared with central or opposing regions (the overall $\mathrm{p}$ value was 0.034 for GOLD I and 0.002 for GOLD II-III and GOLD IV). The selective accumulation of interface DCs was equally present irrespective of the anatomical distribution of the LAs. In total, across the COPD groups more than $80 \%$ of the LA-associated langerin ${ }^{+}$DCs were present within the interface epithelium or in the superficial LA region (exemplified in figures 4A and 5).

The langerin ${ }^{+}$DCs within the alveolar-lymphoid epithelium frequently displayed apical protrusions reaching the alveolar border (figures 4A,B and 5A,C,D). This phenomenon was more frequent in the alveolar-LA epithelium compared with langerin ${ }^{+}$ DCs along the epithelium in the conducting airways. Due to the thin interface at alveolar-LA borders, several DCs were observed with simultaneous physical contact with the alveolar surface and underlying lymphocyte populations within the LAs, including $\mathrm{CD} 20^{+} \mathrm{B}$ cells and $\mathrm{CD} 4^{+} \mathrm{T}$ cells (figure $5 \mathrm{~B}, \mathrm{C}$ ). Other DC populations, such as $\mathrm{CD} 68^{+} \mathrm{CD} 11 \mathrm{c}^{+}$myeloid-like DCs or BDCA $-2^{+}$plasmacytoid DCs did not have similar accumulation at the alveolar interfaces as the langerin ${ }^{+}$DCs (see online supplementary figure S3). 
Simple squamous

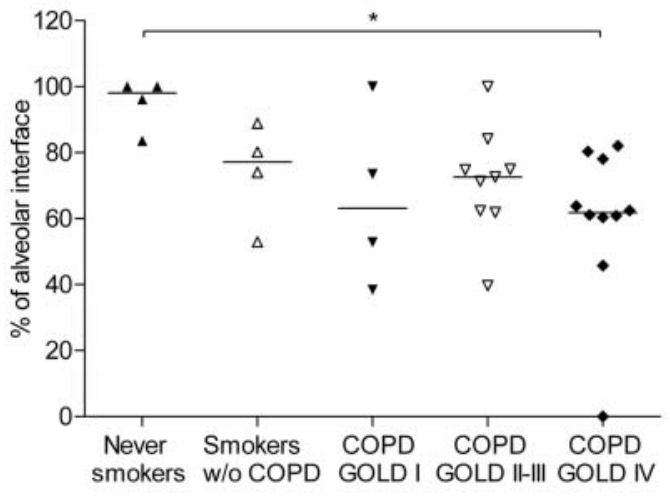

C
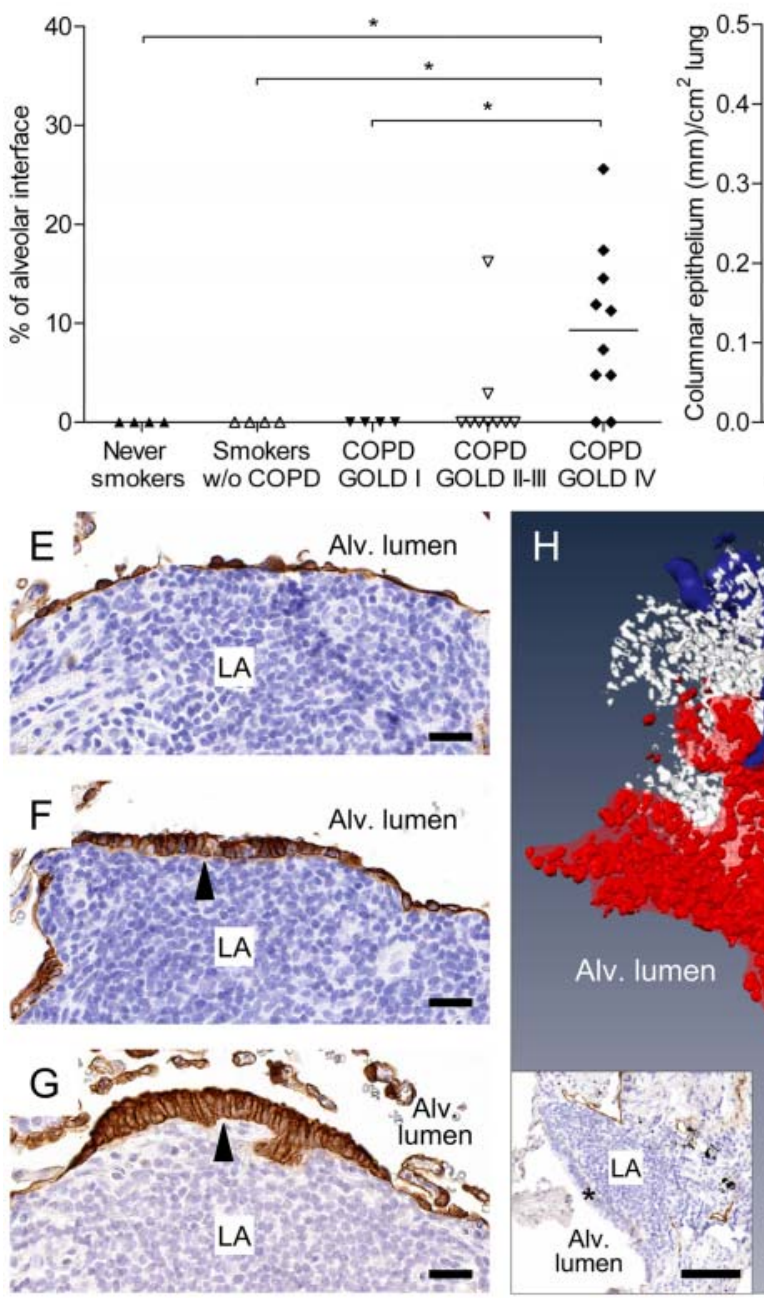

Simple cuboidal

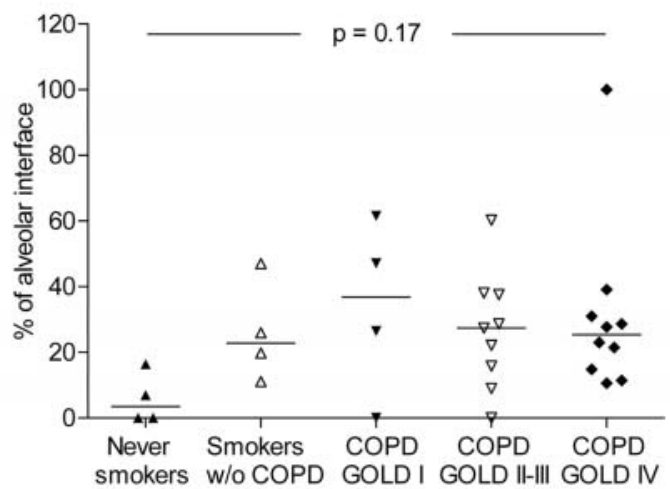

D Total length of columnar epithelium

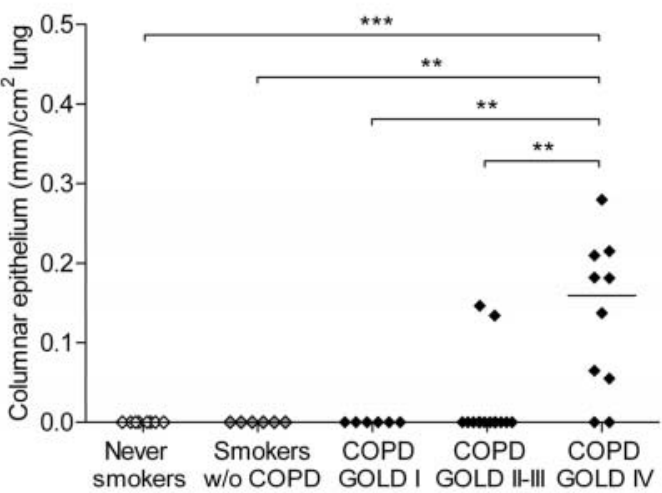

Figure 3 Altered epithelial phenotype at alveolar-lymphoid aggregate (LA) interfaces in patients with chronic obstructive pulmonary disease. The proportion of (A) simple squamous, (B) simple cuboidal and (C) columnar interface epithelium was quantified on podoplanin (lymphatic vessels)/ $\alpha$-smooth muscle actin stained-sections from peripheral lung samples. (D) Quantification of the total length of columnar interface epithelium. (A-D) Statistical analyses were performed using the Kruskal-Wallis non-parametric test followed by Dunn's multiple comparison post test. Horizontal lines indicate medians for each group. ${ }^{*} p<0.05 ;{ }^{*} p<0.01 ;{ }^{* *} p<0.001$. $(E-G)$ Micrographs where brown cytokeratin immunoreactivity visualise the epithelium at alveolar-LA interfaces. Representative images of (E) a simple squamous epithelium; (F) a simple cuboidal epithelium (arrowhead) surrounded by squamous epithelium; $(G)$ a patchy stretch of non-ciliated columnar epithelium (arrowhead, note the sharp border to the flanking squamous epithelium). Cell nuclei were counterstained with Mayer's haematoxylin (blue stain). (H) Computerised three-dimensional rendering of podoplanin-stained sections demonstrated the functional spatial arrangement between the alveolar lumen and interface epithelium (red), the LA (white) and the opposing intertwined network of lymphatic vessels (blue). The inset exemplifies an image from the series with 40 (4 $\mu \mathrm{m}$ thick) podoplanin-stained sections used for the three-dimensional reconstruction (see online supplementary data for methodological details). The asterisk indicates the interface epithelium. Scale bars: $(E-G) 25 \mu \mathrm{m}$; (H, inset) $100 \mu \mathrm{m}$. 


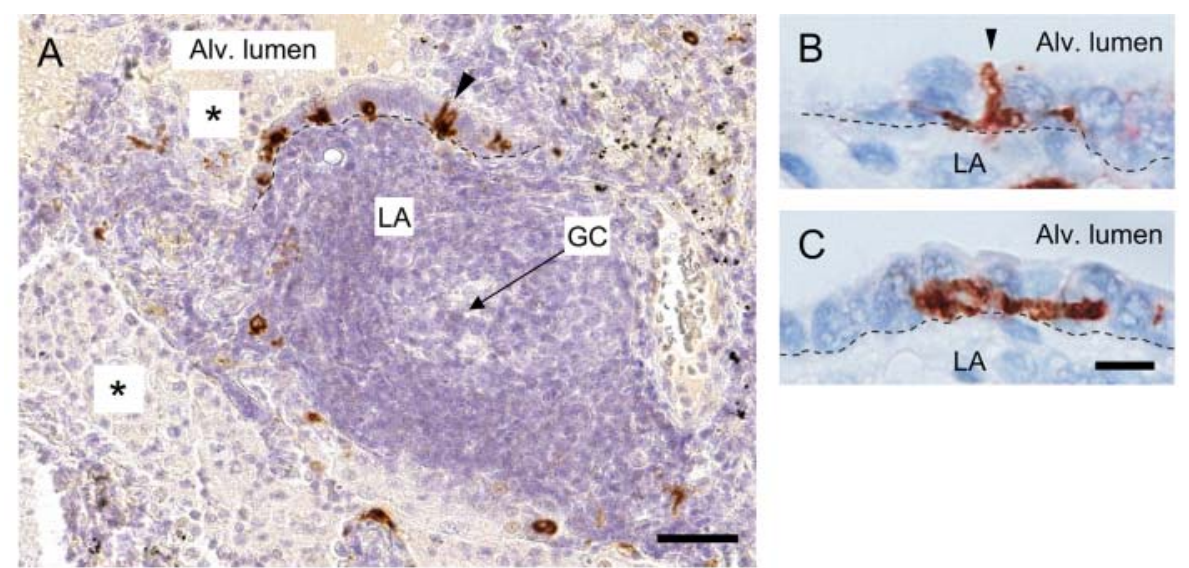



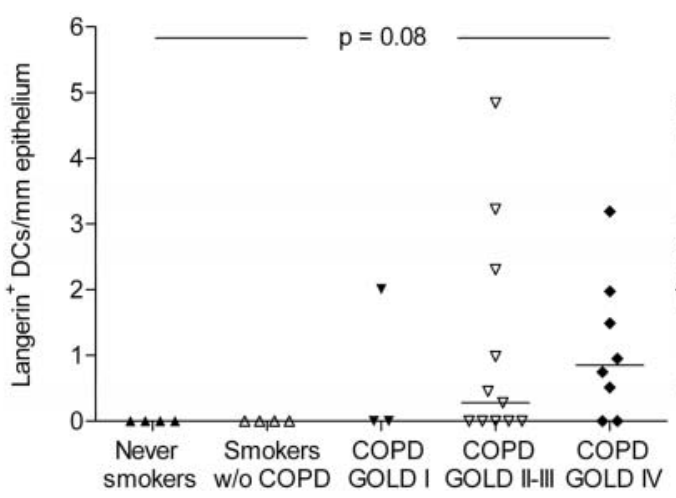

F Total number of interface langerin ${ }^{+} \mathrm{DCs}$

\section{$\mathrm{E}$}
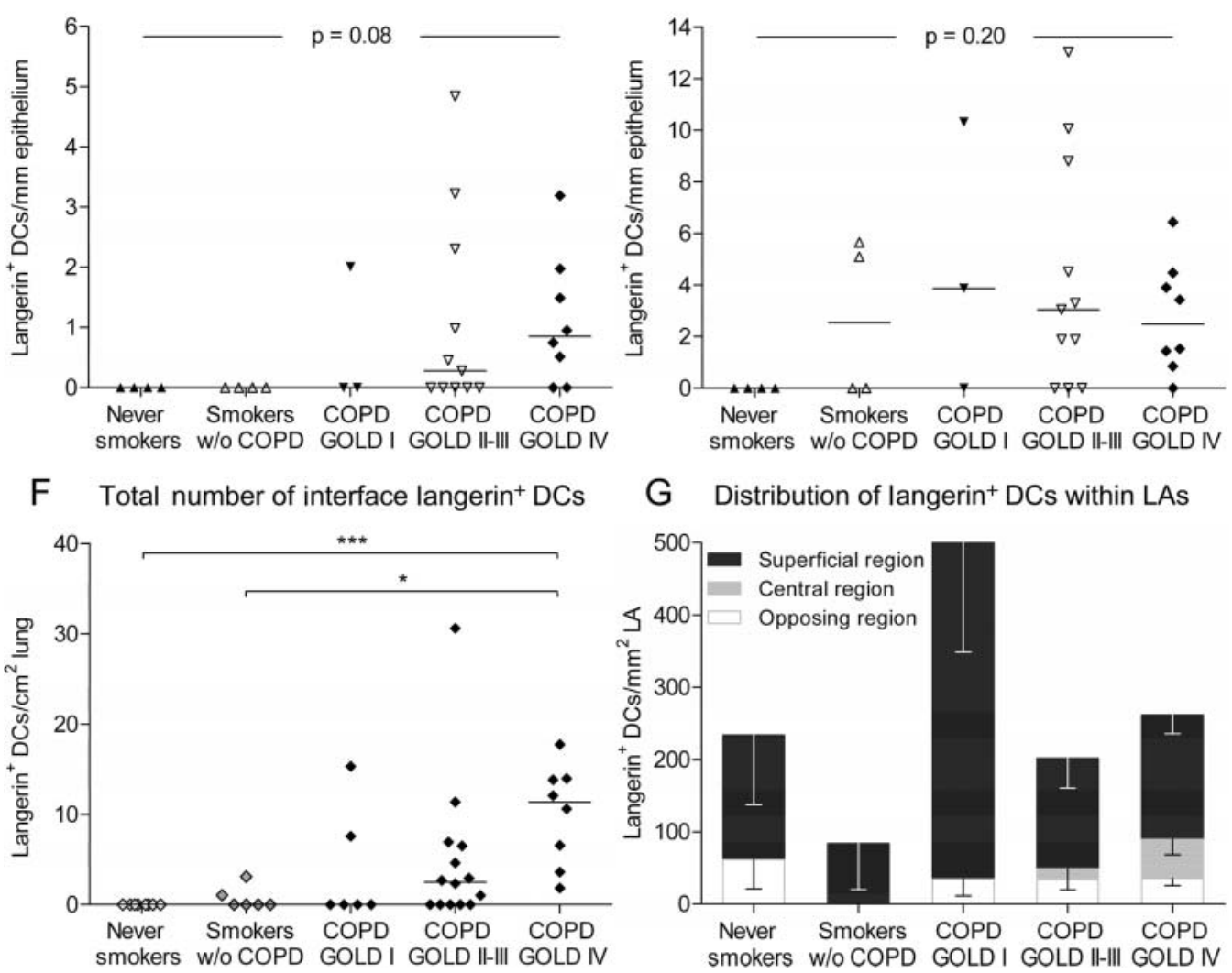

$\mathrm{G}$ Distribution of langerin ${ }^{+} \mathrm{DC}$ s within LAs

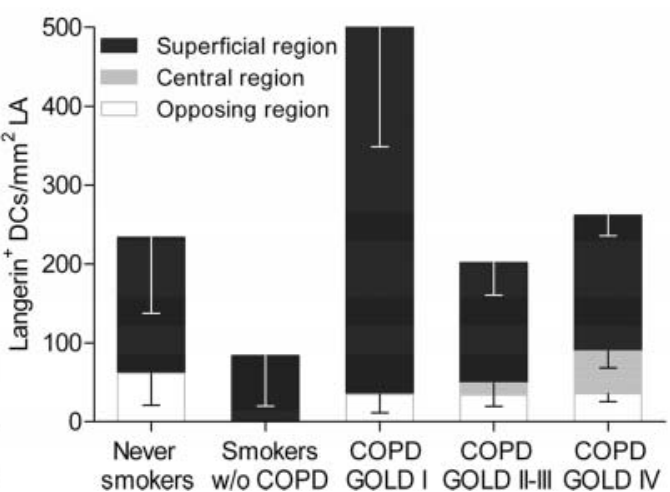

Figure 4 Accumulation of langerin ${ }^{+}$dendritic cells (DCs) along alveolar-lymphoid aggregate (LA) interfaces. (A) Micrograph exemplifying homing of langerin ${ }^{+}$DCs (brown) along the interface epithelium overlying a LA with a germinal centre (GC) (scale bar, $50 \mu \mathrm{m}$ ). Asterisks indicate alveolar lumen filled with macrophages. Arrowhead points to a langerin ${ }^{+} D C$ reaching the alveolar lumen. Black pigment deposition is visible. $(B, C)$ Langerin $^{+}$DCs displayed a typical dendritic morphology with protrusions extending into the alveolar lumen (arrowhead in B) or a more basolateral position (scale bars in $B, C 7 \mu \mathrm{m}$ ). Cell nuclei were counterstained with Mayer's haematoxylin (blue stain). (D,E) Quantification of langerin ${ }^{+}$DCs along peripheral lung LAs with squamous and cuboidal interface epithelium. (F) Quantification of the total number of interface langerin ${ }^{+}$DCs, including DCs in the columnar interface epithelium. (D-F) Statistical analyses were performed using the Kruskal-Wallis non-parametric test followed by Dunn's multiple comparison post test. Horizontal lines indicate medians for each group. ${ }^{*} p<0.05 ;{ }^{* * *} p<0.001$. (G) The densities of langerin ${ }^{+}$ DCs within distinct LA regions. Values are given as mean \pm SEM.

\section{Correlation with lung function parameters}

Several of the parameters associated with the present expansion and remodelling of alveolar-LA interfaces, including the accumulation of interface DCs, correlated inversely with lung function (figure 6 and online supplementary table S2).

\section{DISCUSSION}

The present study reveals new aspects of lung LAs in COPD and supports the emerging notion of the alveolar region as a critical site for induction of adaptive immune responses in inflammatory airway diseases. In light of this, the selective accumulation of langerin ${ }^{+}$DCs at alveolar-LA interfaces in COPD, which represents a major novel finding in this study, is intriguing and suggests an adaptation for increased alveolar antigen uptake. This adaptation is likely to start early during COPD development because interface DCs were observed in relatively mild disease. Although, for obvious reasons, we could not perform functional studies, it seems evident that the present emergence 

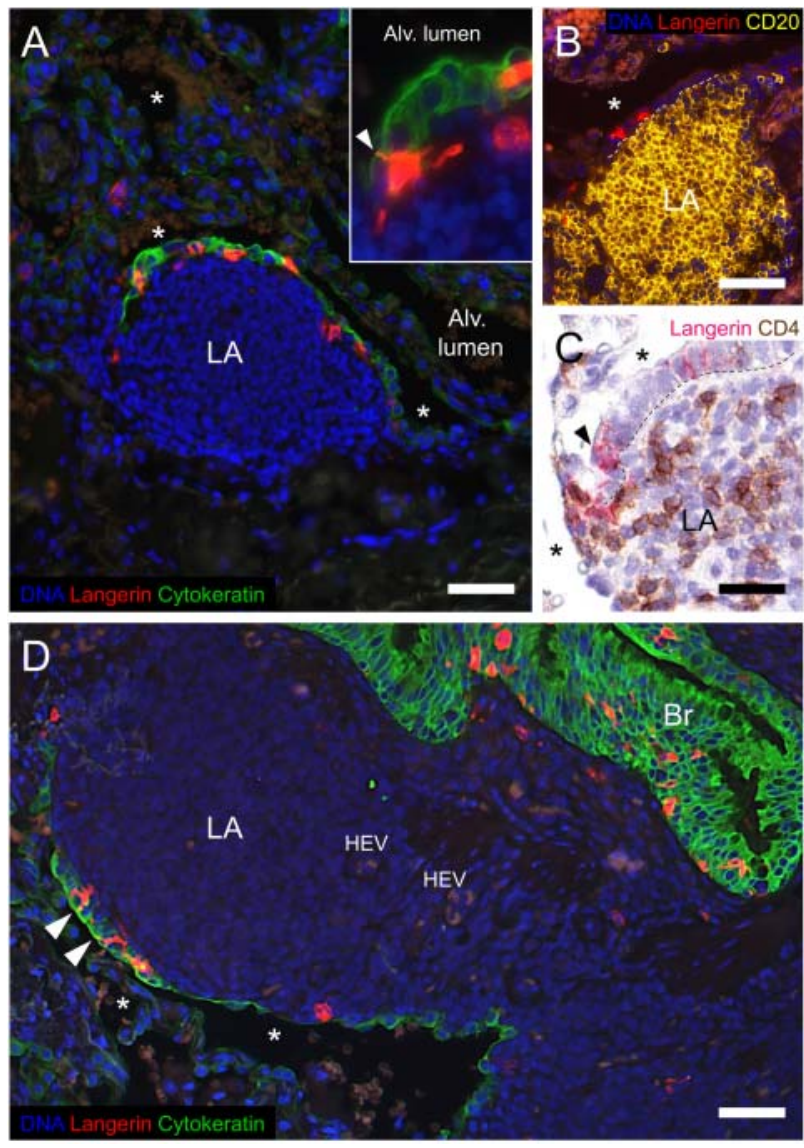

Figure 5 Langerin $^{+}$dendritic cells (DCs) colocalised with cytokeratin ${ }^{+}$ interface epithelium and were in close contact with areas of $B$ and T cells in lymphoid aggregates (LAs). (A) Immunofluorescence double staining revealed colocalisation of langerin ${ }^{+}$DCs (red) and cytokeratin ${ }^{+}$ interface epithelium (green). Langerin ${ }^{+} \mathrm{DCs}$ were in direct contact with (B) $\mathrm{CD}^{2} \mathrm{O}^{+} \mathrm{B}$ cells (yellow) and in close contact with (C) $\mathrm{CD} 4^{+} \mathrm{T}$ cells (brown). (D) A LA with direct interfaces with the bronchiolar (Br) epithelium and the alveolar lumen*. High endothelial venule (HEV)-like structures were frequently observed within the LAs. All sections were counterstained with DNA-binding fluorochrome (Hoechst 3332, blue). In (A-D), asterisks indicate alveolar lumen. In $(A, C, D)$ arrowheads indicate langerin ${ }^{+} D C s$ reaching the alveolar lumen. Scale bars: $(A, B)$ $100 \mu \mathrm{m}$; (C) $25 \mu \mathrm{m}$; (D) $80 \mu \mathrm{m}$.

of DC-rich alveolar-LA interfaces in patients with COPD will result in an increased capacity for faster immune responses in the peripheral lung. Indeed, the intimate spatial relationship between the alveolar lymphoepithelial langerin ${ }^{+}$DCs and the adjacent $\mathrm{T}$ cell areas, and the LAs and extensive network of lymphatic vessels, would create an ideal structural basis for a prompt and highly efficient induction of adaptive immune responses. In this sense the present remodelled LA interface resembles the highly specialised lymphoepithelium overlying the apical part of gut-associated lymphoid tissue (GALT) ${ }^{22}$ or classical BALT structures in bronchi. ${ }^{23}$ Unfortunately, due to a lack of cell-specific markers, we could not study whether $M$ cells, similar to those present in the lymphoepithelium of GALTs, ${ }^{22}$ are also present in the alveolar lymphoepithelium. Theoretically, the occurrence of a higher epithelium at the interfaces could be due to lambertosis (ie, peribronchiolar metaplasia). However, the fact that alveolar columnar epithelium was in the parenchyma exclusively present along the alveolar-lymphoid interfaces suggests that traditional lambertosis is not the major cause of the columnar lymphoepithelium in our study. In any case, regardless of the aetiology of the epithelial changes, it seems clear that the present lymphoepithelium and its selective accumulation of langerin ${ }^{+}$DCs represent a novel arena for alveolar antigen uptake. Apart from the release of DC-activating mediators by epithelial cells, ${ }^{24}$ physical DC-epithelial interactions are needed to maintain the epithelial barrier when DCs protrude across the tight junctions. ${ }^{25}$ Whether this types of interactions are increased in the columnar interface epithelium remains to be established.

Our study also provides the first quantitative data to show that the LAs in COPD are present in all major anatomic regions of the peripheral lung. In agreement, previous studies exploring lung LAs in COPD demonstrated that, apart from BRALTs, LAs were also increased in the alveolar parenchyma. ${ }^{12-14}$ However, it has previously been speculated that such seemingly alveolar LAs have physical contact with airways or pulmonary vessels in their three-dimensional in vivo context. Using three-dimensional reconstructions, this study thus proves for the first time that true alveolar-only LAs exist and shows that in COPD these represent around $25 \%$ of the total lung LAs. Moreover, this study provides novel quantitative data showing that irrespective of anatomical distribution, the vast majority of the total LAs in COPD lungs have direct interfaces towards the alveolar lumen. Thus, BRALTs and VALTs should conceptually also be viewed as alveolar associated (ie, ALTs). This new view of LAs agrees with previous findings in COPD lungs. For example, van der Strate and colleagues ${ }^{12}$ reported that BRALTs very rarely reached the bronchiolar epithelium. In agreement with our study, a similar adventitial distribution has also been noted for VALTs. ${ }^{26} 27$ Collectively, it thus seems that an extensive interface towards the alveolar lumen is a general feature of all types of lung LAs.

The origin of the antigens driving the LA formation in COPD is still debated. ${ }^{11}$ One source of peripheral antigens comes from lower respiratory tract infections. Apart from the exacerbation-associated infections in $\mathrm{COPD},{ }^{15}$ recent microbiome studies reveal a chronic and low-grade presence of lung pathogens outside exacerbations. ${ }^{28}$ In addition, inhaled immunogenic microparticles may also induce alveolar adaptive immunity in COPD, as indicated by LA formation in pathogenfree mice exposed to cigarette smoke. ${ }^{12}$ Several studies have also demonstrated elevated levels of antibodies to self-antigens. ${ }^{29} 30$ Taken together, the antigens captured by the alveolar interface DCs revealed in this study should be expected to be multifaceted. Although the immune responses initiated at alveolar-LA interfaces are likely to have beneficial host defence purposes, it is also likely that in severe COPD these may turn into pathogenic events. It can thus be surmised that, apart from any autoimmune components in COPD, a non-proportional expansion of peripheral alveolar-LA interfaces at advanced stages of COPD results in an 'immunological hyperreactivity' that may evoke harmful and exaggerated immune responses to the frequent infections that hit this patient category.

As for most invasive patient studies, a potential weakness with our study was the limited number of subjects. In part this was compensated by the fact that several separated lung regions (each containing multiple anatomic compartments) were analysed from each patient. In any case, despite restricted patient numbers, robust changes could be observed for the main parameters. In the present study the excised lung samples were immersed into a fixative rather than using a perfusion fixation approach. Although this may result in tissue samples with different degrees of compression, the key parameters in the present study, such as the quantitative analysis of epithelial alterations and degree of DC infiltration along the alveolar-lymphoid 
Figure 6 Correlations. There was a negative relationship between forced expiratory volume in $1 \mathrm{~s}\left(\mathrm{FEV}_{1}\right) \%$ of predicted and $(\mathrm{A})$ the total tissue immunoreactivity for CD20, (B) the total length of alveolar interfaces, (C) the total length of columnar interface epithelium, and (D) the total number of interface langerin ${ }^{+}$dendritic cells. Black symbols, lines and text represent patients with Global Initiative for Chronic Obstructive Lung Disease (GOLD) stage I-IV chronic obstructive pulmonary disease (COPD). Grey symbols represent smokers without COPD. White symbols represent never smokers. Red lines and text represent all study groups. Spearman's rank correlation was used.

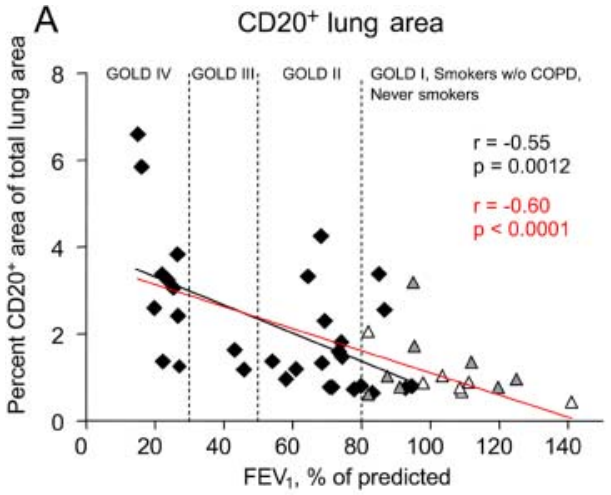

B Total length of alveolar interfaces

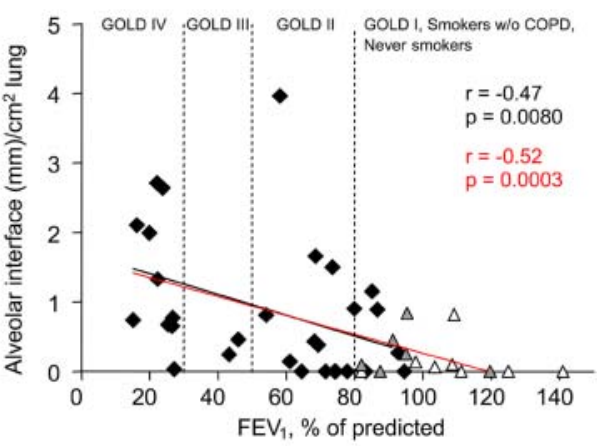

C Total length of columnar epithelium

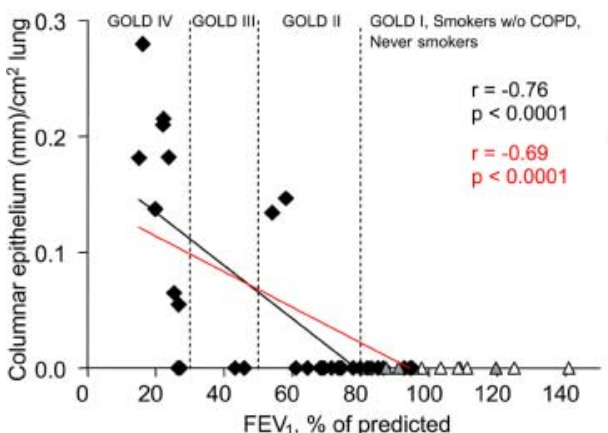

interface, are independent of the degree of compression. Also, it cannot be excluded that suspected cancer in several of our cohorts has influenced the immunological status of the lung. However, since we only included subjects with well delineated bronchial tumours and restricted the analyses to regions far away from the tumour, we believe that any such influence is minimal. Furthermore, the most striking findings were found in patients with GOLD stage IV disease who did not have cancer.

The present findings are expected to have a significant bearing on many other diseases characterised by peripheral lung LAs, for example, long-term distal infections, ${ }^{31} 32$ cystic fibrosis ${ }^{33}$ and idiopathic pulmonary fibrosis. ${ }^{32}$ Notably, patients with autoimmune diseases like idiopathic pulmonary arterial hypertension ${ }^{27}$ or rheumatoid arthritis with pulmonary complications ${ }^{32}$ develop ectopic lung LAs. Further work is needed to establish if emergence of the DC-rich alveolar-LA interfaces revealed in this study is a general feature of all diseases characterised by lung LAs.

This study may also have important implications for the growing field of pulmonary vaccinology. Respiratory pathogens enter the body at mucosal or alveolar surfaces, but systemically delivered vaccines fail to induce a therapeutic protective response at these sites. This has created a rational for local pulmonary delivery of vaccines to increase lung protection and systemic immunity. ${ }^{34}$ The novel type of DC-rich alveolar-LA interface shown in this study would likely contribute to an increased efficacy of new and emerging pulmonary vaccine strategies. $^{36} 37$

In summary, this study shows that severity of COPD is associated with a marked remodelling of alveolar-LA interfaces. These alterations, which involve a selective accumulation of interface DCs and correlate with lung function, predict an increased capacity to respond to alveolar antigens and may thus contribute to aggravated inflammation in COPD.

Acknowledgements We thank Karin Jansner and Britt-Marie Nilsson for skilful technical assistance with tissue processing and serial sectioning.
Contributors MM collected tissue samples, performed laboratory work, analysed the data and wrote the manuscript. CKA contributed to tissue handling and sample characterisation. KAS performed three-dimensional reconstructions. PG participated in early preliminary experiments. CGL contributed to the clinical characterisation. JSE designed and supervised the study and wrote the manuscript. All authors participated in manuscript editing and approved the final version of the manuscript.

Funding This work was supported by the Swedish Heart and Lung Foundation and the Swedish Medical Research Council.

Competing interests None.

Ethics approval Ethics approval was provided by the Swedish Research Ethics Committee in Lund.

Provenance and peer review Not commissioned; externally peer reviewed.

\section{REFERENCES}

1 Rabe KF, Hurd S, Anzueto A, et al. Global strategy for the diagnosis, management, and prevention of chronic obstructive pulmonary disease: GOLD executive summary. Am J Respir Crit Care Med 2007:176:532-55.

2 Cosio MG, Saetta M, Agusti A. Immunologic aspects of chronic obstructive pulmonary disease. N Engl J Med 2009:360:2445-54.

3 Hogg JC. Pathophysiology of airflow limitation in chronic obstructive pulmonary disease. Lancet 2004;364:709-21.

4 Brusselle GG, Joos GF, Bracke KR. New insights into the immunology of chronic obstructive pulmonary disease. Lancet 2011;378:1015-26.

5 Jahnsen FL, Strickland DH, Thomas JA, et al. Accelerated antigen sampling and transport by airway mucosal dendritic cells following inhalation of a bacterial stimulus. J Immunol 2006;177:5861-67.

6 Vermaelen KY, Carro-Muino I, Lambrecht BN, et al. Specific migratory dendritic cells rapidly transport antigen from the airways to the thoracic lymph nodes. J Exp Med 2001;193:51-60.

7 Hogg JC, Chu F, Utokaparch S, et al. The nature of small-airway obstruction in chronic obstructive pulmonary disease. N Engl J Med 2004;350:2645-53.

8 Pabst R, Gehrke I. Is the bronchus-associated lymphoid tissue (BALT) an integral structure of the lung in normal mammals, including humans? Am J Respir Cell Mol Biol 1990;3:131-5.

9 Aloisi F, Pujol-Borrell R. Lymphoid neogenesis in chronic inflammatory diseases. Nat Rev Immunol 2006;6:205-17.

10 Carragher DM, Rangel-Moreno J, Randall TD. Ectopic lymphoid tissues and local immunity. Semin Immunol 2008;20:26-42.

11 Brusselle GG, Demoor T, Bracke KR, et al. Lymphoid follicles in (very) severe COPD: beneficial or harmful? Eur Respir J 2009;34:219-30. 
12 van der Strate BW, Postma DS, Brandsma CA, et al. Cigarette smoke-induced emphysema: a role for the B cell? Am J Respir Crit Care Med 2006;173:751-8.

13 Plumb J, Smyth LJ, Adams HR, et al. Increased T-regulatory cells within lymphocyte follicles in moderate COPD. Eur Respir J 2009;34:89-94.

14 Polverino F, Baraldo S, Bazzan E, et al. A novel insight into adaptive immunity in chronic obstructive pulmonary disease: $\mathrm{B}$ cell activating factor belonging to the tumor necrosis factor family. Am J Respir Crit Care Med 2010;182:1011-19.

15 Sethi S, Murphy TF. Infection in the pathogenesis and course of chronic obstructive pulmonary disease. N Engl J Med 2008;359:2355-65.

16 Thornton EE, Looney MR, Bose 0 , et al. Spatiotemporally separated antigen uptake by alveolar dendritic cells and airway presentation to T cells in the lung. J Exp Med 2012:209:1183-99.

17 Veres TZ, Voedisch S, Spies E, et al. Spatiotemporal and functional behavior of airway dendritic cells visualized by two-photon microscopy. Am J Pathol 2011;179:603-9.

18 Andersson CK, Mori M, Bjermer L, et al. Alterations in lung mast cell populations in patients with chronic obstructive pulmonary disease. Am J Respir Crit Care Med 2010;181:206-17

19 Skaland I, Nordhus M, Gudlaugsson E, et al. Evaluation of 5 different labeled polymer immunohistochemical detection systems. Appl Immunohistochem Mol Morphol 2010;18:90-6.

20 Demedts IK, Bracke KR, Van Pottelberge G, et al. Accumulation of dendritic cells and increased CCL20 levels in the airways of patients with chronic obstructive pulmonary disease. Am J Respir Crit Care Med 2007;175:998-1005.

21 Van Pottelberge GR, Bracke KR, Demedts IK, et al. Selective accumulation of Langerhans-type dendritic cells in small airways of patients with COPD. Respir Res 2010;11:35.

22 Corr SC, Gahan CC, Hill C. M-cells: origin, morphology and role in mucosal immunity and microbial pathogenesis. FEMS Immunol Med Microbiol 2008:52:2-12.

23 Randall TD. Bronchus-associated lymphoid tissue (BALT) structure and function. Adv Immunol 2010;107:187-241.

24 Hammad H, Lambrecht BN. Dendritic cells and epithelial cells: linking innate and adaptive immunity in asthma. Nat Rev Immunol 2008;8:193-204.
25 Blank F, Wehrli M, Lehmann A, et al. Macrophages and dendritic cells express tight junction proteins and exchange particles in an in vitro model of the human airway wall. Immunobiology 2011;216:86-95.

26 Olloquequi J, Montes JF, Prats A, et al. Significant increase of CD57+ cells in pulmonary lymphoid follicles of COPD patients. Eur Respir J 2011:27:289-98.

27 Perros F, Dorfmuller $\mathrm{P}$, Montani $\mathrm{D}$, et al. Pulmonary lymphoid neogenesis in idiopathic pulmonary arterial hypertension. Am J Respir Crit Care Med 2012;185:311-21.

28 Sze MA, Dimitriu PA, Hayashi $\mathrm{S}$, et al. The lung tissue microbiome in chronic obstructive pulmonary disease. Am J Respir Crit Care Med 2012;185:1073-80.

29 Feghali-Bostwick CA, Gadgil AS, Otterbein LE, et al. Autoantibodies in patients with chronic obstructive pulmonary disease. Am J Respir Crit Care Med 2008;177:156-63.

30 Lee SH, Goswami S, Grudo A, et al. Antielastin autoimmunity in tobacco smoking-induced emphysema. Nat Med 2007;13:567-9.

31 Suda T, Chida K, Hayakawa $H$, et al. Development of bronchus-associated lymphoid tissue in chronic hypersensitivity pneumonitis. Chest 1999;115:357-63.

32 Rangel-Moreno J, Hartson L, Navarro C, et al. Inducible bronchus-associated lymphoid tissue (BBALT) in patients with pulmonary complications of rheumatoid arthritis. J Clin Invest 2006;116:3183-94.

33 Hubeau C, Lorenzato M, Couetil JP, et al. Quantitative analysis of inflammatory cells infiltrating the cystic fibrosis airway mucosa. Clin Exp Immunol 2001;124:69-76.

34 Chen K, Cerutti A. Vaccination strategies to promote mucosal antibody responses. Immunity 2010;33:479-91.

35 Vujanic A, Wee JL, Snibson KJ, et al. Combined mucosal and systemic immunity following pulmonary delivery of ISCOMATRIX adjuvanted recombinant antigens. Vaccine 2010;28:2593-7.

36 Nembrini C, Stano A, Dane KY, et al. Nanoparticle conjugation of antigen enhances cytotoxic T-cell responses in pulmonary vaccination. Proc Natl Acad Sci USA 2011;108:E989-97.

37 Pabst R, Tschernig T. Bronchus-associated lymphoid tissue: an entry site for antigens for successful mucosal vaccinations? Am J Respir Cell Mol Biol 2010;43:137-41. 